\title{
ANÁliSE REOLÓGICA DA SOLUÇÃO AQUOSA DE CORANTE DE JENIPAPO (Genipa americana L.)
}

\author{
E. L. ANDRADE ${ }^{1}$, M. M. MATSUNAGA ${ }^{2}$, C. M. L. $\operatorname{COSTA}^{2}$ e L. J. G. de FARIA ${ }^{2}$ \\ ${ }^{1}$ Universidade Federal do Pará - Programa de Pós-Graduação em Engenharia de Recursos \\ Naturais da Amazônia \\ ${ }^{2}$ Universidade Federal do Pará - Faculdade de Engenharia Química \\ E-mail para contato: elisangela@ufpa.br
}

RESUMO - Realiza-se uma caracterização reológica de uma solução aquosa de corante de jenipapo (Genipa amerina L.) em pó, diluído nas proporções de 5, 10 e 15\%, utilizando um viscosímetro rotacional acoplado a um sistema de aquisição de dados, nas temperaturas 25,35 e $45^{\circ} \mathrm{C}$. O estudo reológico de suspensões está diretamente ligado ao controle de qualidade do produto (intermediário e final), no modo de armazenamento, transporte e na verificação do prazo de validade. O corante de jenipapo é extraído do endocarpo do fruto em estádio verde maturação, e quando em contato com o ar escurece gradativamente, adquirindo uma coloração azul intensa. A análise dos dados de 12 corridas experimentais, estatisticamente planejadas, indica que a suspensão (corante-água) apresenta comportamento de um fluido nãonewtoniano e pseudoplástico, podendo ser adequadamente descrito pelo modelo de Sisko (1958).

\section{INTRODUÇÃO}

O jenipapeiro pertence a uma família que ocupa o quarto lugar de todo o reino vegetal em número de espécies, a família Rubiceae (Chuquieri et al.,2004). Apresenta importância econômica, tanto como essência florestal quanto como produtora de frutos utilizados na alimentação humana (Barros, 1970).

O comportamento reológico de alimentos vem sendo estudado por vários autores. Haminiuk et al. (2006) avaliaram o comportamento reológico de amora-preta na faixa de $10^{\circ} \mathrm{C}$ a $60^{\circ} \mathrm{C}$, constatando um comportamento pseudoplástico. Constataram que o aumento da temperatura provoca uma diminuição na viscosidade aparente e do índice de consistência, conforme esperado para polpa de fruta. O mesmo comportamento foi encontrado por Guerrero e Alzamora (1997) ao avaliarem o comportamento reológico de purê de banana.

A crescente necessidade e procura dos parâmetros reológicos para os diversos fluidos manipulados nas indústrias de processamento está ligada também à grande importância econômica que estes fluidos e equipamentos de manipulação representam. Essas propriedades são úteis não só como medida de qualidade, segundo Pelegrine et al., (2000), mas também no projeto dos processos de industrialização tais como bombeamento, agitação, transporte em 
tubulações, evaporação, pasteurização, resfriamento e congelamento. Assim, por meio de dados de tensão de cisalhamento e taxa de deformação em três diferentes temperaturas e três diferentes concentrações, tem-se como finalidade determinar as propriedades reológicas em função da temperatura e concentração do corante total de jenipapo verde em pó. Oliveira (1997) avaliou o comportamento desse corante pó como matéria prima para a indústria de tintas (pó de revestimento - poliéster). A análise dos resultados demonstra que ocorrerem variações nos sistemas onde é adicionado o corante de jenipapo, inadmissíveis para revestimentos, não sendo, portanto recomendado como corante para revestimentos orgânicos. Dessa forma, o objetivo deste trabalho é realizar uma caracterização reológica de uma solução aquosa de corante de jenipapo (Genipa amerina L.) em pó, para determinação de parâmetros de qualidade que viabilizem sua utilização na indústria de alimentos, cosméticos e fármacos.

\section{MATERIAIS E MÉTODOS}

O presente experimento é conduzido no laboratório Engenharia de Produtos Naturais LEPRON, pertencente à Faculdade de Engenharia Química - UFPA - Campus Belém.

\subsection{Matéria-prima}

São utilizados frutos de Jenipapo (Genipa americana L.) verdes (pois uma vez maduros são inadequados à obtenção do corante), coletados no campus básico da UFPA em Belém - PA, variedade sempre-florens. Lavam-se os frutos com sabão neutro e escova em água corrente, pesam-se cada fruto, em seguida extrai-se o endocarpo e congela-se a $-18^{\circ} \mathrm{C}$ a fim de conservá-lo, na temperatura ideal para retardar a maturação do fruto até o prosseguimento do trabalho. Na Figura 1 (A) têm-se um fruto de jenipapo verde seccionado ao meio, apresentando o endocarpo oxidado ao ar livre. A Figura 1 (B) consiste de uma amostra do corante de jenipapo verde em pó, onde se observa a forte coloração azul marinho.
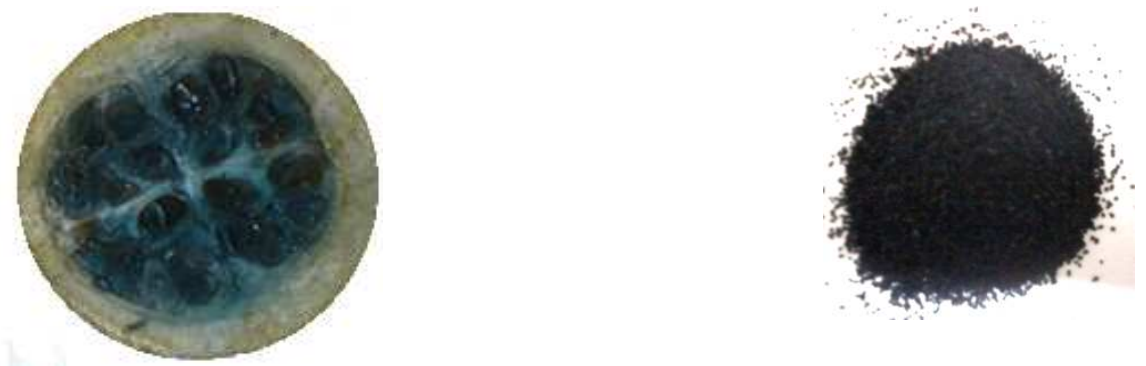

Figura1 - Fruto de jenipapo verde com o endocarpo oxidado (A) e Amostra de corante de jenipapo em pó (B) 


\subsection{Obtenção do corante total em pó}

O processo de obtenção do corante total de jenipapo verde, pelo processo de oxidação induzida, consiste na remoção do endocarpo dos frutos, submetendo-se o material a um processo de secagem a $70^{\circ} \mathrm{C}$ durante 24 horas em secador de bandejas, onde o processo de oxidação é acelerado, proporcionando o aparecimento do corante azul. Em seguida reduz-se o tamanho da amostra em moinho de facas, para a obtenção do pó, conforme fluxograma ilustrado na Figura 2.

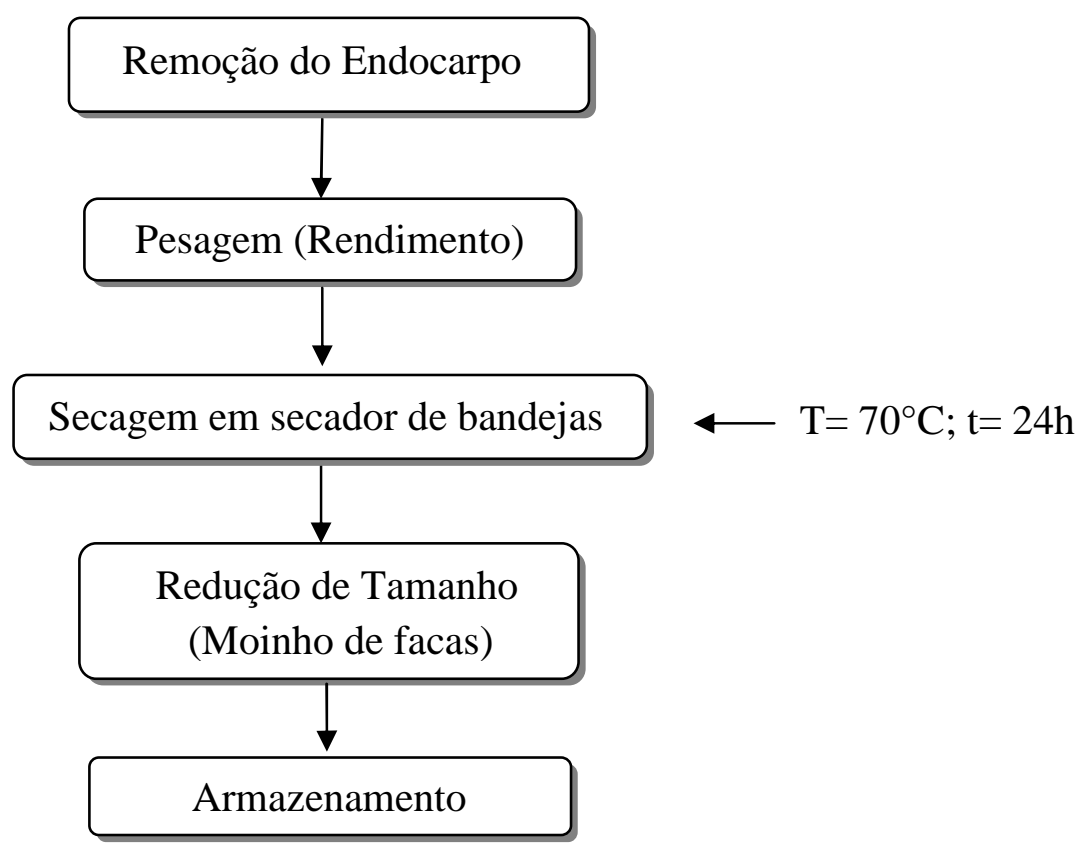

Figura 2 - Fluxograma da obtenção do corante total de jenipapo verde

\subsection{Preparo da suspensão}

Prepara-se uma suspensão aquosa de corante (total) em pó de jenipapo nas proporções de 5,10 e $15 \%$ de corante em pó, utilizando-se homogeneizador de alta eficiência - Ultra Turrax T25 - IKA com rotação de $24.500 \mathrm{rpm}$. 


\subsection{Viscosidade aparente}

A medida das viscosidades aparentes das suspensões aquosas do corante é realizada nas temperaturas de 25,35 e $45^{\circ} \mathrm{C}$ à pressão ambiente, utilizando-se um viscosímetro Haake modelo Viscotester $6^{\mathrm{L}}$, com o splindle L4, considerado o mais adequado para esta análise, nas velocidades de rotação 10, 12, 20, 30, 50, 60, 100 e $200 \mathrm{rpm}$. Um banho criostático de recirculação Haake F3, é acoplado ao equipamento para que as amostras se mantenham na temperatura desejada. A aquisição dos dados é feita por meio do software RheoWin.

\subsection{Modelos reológicos}

Calculam-se as viscosidades, tensões de cisalhamento e as taxas de deformação com os dados experimentais obtidos no viscosímetro por meio do software RheoWin, para as diferentes temperaturas e velocidades de rotação, utilizando-se a metodologia de Mitschka (1982). Ajustam-se os modelos de Sisko (Equação 1), Ostwald-de-Waele (Equação 2) e Casson (Equação 3), aos pontos experimentais de tensão de cisalhamento em função da taxa de deformação para descrever o comportamento reológico da suspensão, por meio software Statistica versão 7.0 (2004).

$$
\begin{array}{rc}
\text { Sisko: } & \tau=\tau_{y}+\eta_{p} \dot{\gamma} \\
\text { Ostwald-de-Waele: } & \tau=K \dot{\gamma}^{n} \\
\text { Casson: } & \tau^{0,5}=\mathrm{K}_{0 C}+\mathrm{K}_{C} \dot{\gamma}^{0,5}
\end{array}
$$

Verifica-se a qualidade do ajuste com base nos pressupostos estatísticos: coeficiente de determinação múltipla $\left(\mathrm{R}^{2}\right)$ e erro médio estimado (SE), dado pela Equação 4. Onde y e $\hat{y}$ correspondem aos valores observados experimentalmente e calculados pelo modelo proposto, respectivamente, e $\mathrm{n}$ representa o número de observações.

$$
S E=\sqrt{\frac{\sum_{i=1}^{n}(y-\hat{y})^{2}}{G R L}}
$$

Onde SE é o erro médio estimado (decimal) e GRL corresponde ao grau de liberdade do modelo (número de observações menos o número de parâmetros do modelo). 


\section{RESULTADOS E DISCUSSÃO}

Dos três modelos testados para ajustar os dados experimentais de tensão de cisalhamento em função da taxa de deformação da suspensão corante, somente o modelo de Sisko (1958) consegue explicar mais de $94 \%$ das variabilidades experimentais e apresenta resíduos independentes e com distribuição aleatória com os valores preditos de tensão de cisalhamento, além de apresentar homogeneidade de variâncias.

Na Tabela 1 constam os parâmetros do modelo de Sisko (1958) conforme os valores de temperatura e concentração da suspensão corante, bem como as estatísticas $\mathrm{R}^{2}$, P e SE, que demonstram a qualidade do ajuste proposto.

Tabela 1 - Parâmetros do modelo de Sisko (1958) ajustado aos pontos experimentais de tensão versus deformação para o corante de jenipapo em função da concentração e temperatura

\begin{tabular}{|c|c|c|c|c|c|c|}
\hline \multirow{2}{*}{$\mathrm{T}\left({ }^{\circ} \mathrm{C}\right)$} & \multirow{2}{*}{$\mathrm{C}(\%)$} & \multicolumn{3}{|c|}{ Parâmetros } & \multirow{2}{*}{$\mathrm{R}^{2}$} & \multirow{2}{*}{$\mathrm{SE}$} \\
\cline { 3 - 7 } & & $\mathrm{a}$ & $\mathrm{b}$ & $\mathrm{n}$ & & \\
\hline \multirow{3}{*}{25} & 5 & $-4,45312$ & 4,94886 & 0,996890 & 0,9484 & 0,6673 \\
\cline { 2 - 7 } & 10 & $-8,28047$ & 8,594688 & 0,994513 & 0,9862 & 0,5233 \\
\cline { 2 - 7 } & 15 & $-0,211396$ & $-1,176548$ & 0,777164 & 0,9991 & 0,5460 \\
\hline \multirow{3}{*}{35} & 5 & $-0,026529$ & 0,268678 & 0,703594 & 0,9560 & 0,9990 \\
\cline { 2 - 7 } & 10 & $-0,108752$ & 0,527384 & 0,759901 & 0,9967 & 0,8526 \\
\cline { 2 - 7 } & 15 & $-1,23956$ & 2,629478 & 0,879538 & 0,9677 & 0,5930 \\
\hline \multirow{3}{*}{45} & 5 & $-0,017498$ & 0,314189 & 0,588745 & 0,9778 & 0,7688 \\
\cline { 2 - 7 } & 10 & 0,054563 & 2,049311 & 0,036031 & 0,9456 & 0,5510 \\
\cline { 2 - 7 } & 15 & 0,045279 & 2,192152 & 0,296692 & 0,9888 & 0,5289 \\
\hline
\end{tabular}

Os valores do índice $\mathrm{n}$ menores que 1 , caracteriza comportamento de fluido nãoNewtoniano e pseudoplástico.

A Figura 1 consiste de um reograma para a suspensão de corante de jenipapo a $25^{\circ} \mathrm{C}$ nas concentrações 5, 10 e 15\%. A Figura 2 ilustra o reograma para a suspensão do corante $15 \%$ nas temperaturas 25,35 e $45^{\circ} \mathrm{C}$. Verifica-se pelo comportamento das curvas das Figuras 1 e 2, que mantendo-se constante uma taxa de deformação e verificando o valor correspondente da tensão, a viscosidade aparente da suspensão aumenta com a concentração e diminui sensivelmente com a temperatura, considerando-se a viscosidade aparente responsável pela resistência ao escoamento. 


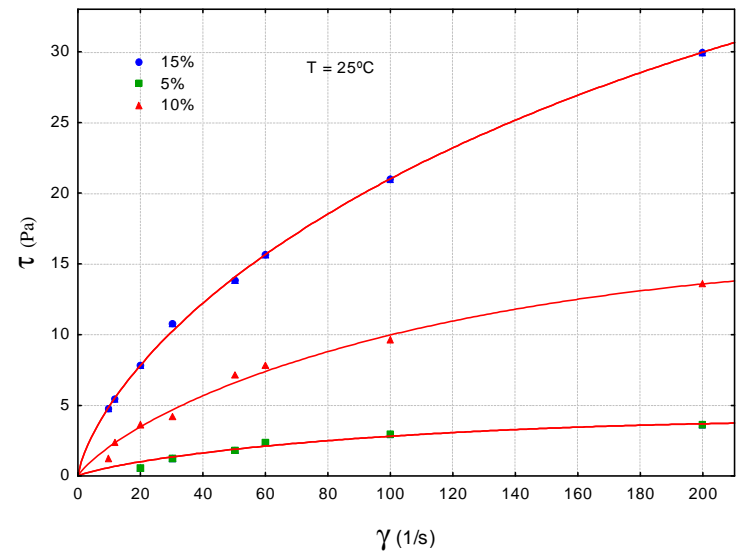

Figura 1 - Curvas de tensão de cisalhamento versus taxa de deformação para o corante de jenipapo a $25^{\circ} \mathrm{C}$ nas concentrações 5,10 e $15 \%$

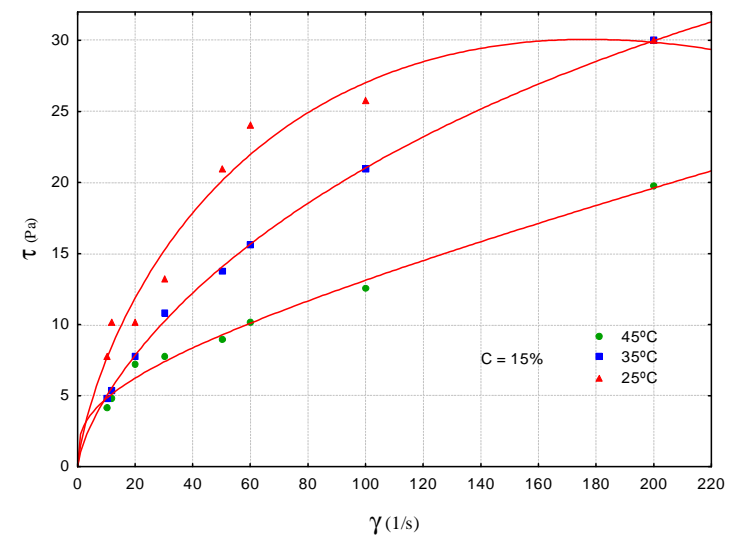

Figura 2 - Curvas de tensão de cisalhamento versus taxa de deformação para o corante de jenipapo a $15 \%$ nas temperaturas 25,35 e $45^{\circ} \mathrm{C}$

\section{CONCLUSÕES}

Os resultados deste trabalho demonstram que ocorre uma efetiva variação na tensão de cisalhamento da suspensão aquosa com o aumento da concentração de corante de jenipapo em pó à temperatura constante, o que implica no aumento da viscosidade aparente. Com o aumento da temperatura a viscosidade aparente da suspensão decresce, mantendo-se constante a concentração da suspensão. Esses resultados podem nortear aplicações do corante de jenipapo em processos de recobrimento de produtos agrícolas na cor azul, na encapsulação do corante por atomização, na elaboração de cosméticos ou na especificação de máquinas de fluxo para o transporte da suspensão.

\section{NOMENCLATURA}

$\tau$ - tensão de cisalhamento $(\mathrm{Pa})$

$\dot{\gamma}$ - taxa de deformação $\left(\mathrm{s}^{-1}\right)$

$\tau_{y}$ - índice de consistência $\left(\mathrm{Pa} . \mathrm{s}^{\mathrm{n}}\right)$

$\eta_{p}$ - viscosidade plástica (Pa.s)

$\mathrm{K}$ - índice de consistência (Pa.s ${ }^{\mathrm{n}}$ )

$\mathrm{n}$ - índice de comportamento do fluido

$\mathrm{K}_{0 C}$ - tensão de cisalhamento inicial $(\mathrm{Pa})$ 
$\mathrm{K}_{C}$ - viscosidade plástica de Casson (Pa.s)

$\mathrm{SE}$ - erro médio estimado

$\mathrm{n}$ - tamanho da amostra

C- concentração da suspensão corante (\%)

$\mathrm{T}$ - temperatura $\left({ }^{\circ} \mathrm{C}\right)$

$\mathrm{R}^{2}$ - coeficiente de determinação múltipla

\section{REFERÊNCIAS}

BARROS, R.C. Jenipapeiro. F. Flor, n.4, v.18, p.1-3, 1970.

CASSON, N. A flow equation for pigment-oil suspensions of the printingink type, in Rheology of disperses suspensions, C. C. Mill (ed), (Pergamon Press, New York). 1959. $348 p$.

CHUQUIERI, A.; DI MAIO, F. R.; PEIXOTO, A.L. A distribuição geográfica da família Rubiceae Juss. na flora brasiliense de Martius. Rodriguésia, Rio de Janeiro, v.55, n.84, p.4757, 2004.

GUERRERO, S. N.; ALZAMORA, S. M. Effect of pH, Temperature and Glucose Addition on Flow Behavior of Fruit Pur'ees I. Banana Pur'ee. Journal of Food Engineering. v. 33, p. 239-256, 1997.

HAMINIUK, C.W.I.; SIERAKOWSKI, M.R.; IZIDORO, D.R.; MASSON, M. L. Rheological characterization of blackberry pulp. Brazilian Journal of Food Technology, Campinas, v.9, n.4, p.291-296, 2006.

LABA, D. Rheological properties of cosmetics and toiletries. New York: Marcel Dekker, p. 09-33, 1993.

LEONARDI, G.R.; MAIA CAMPOS, P.M.B.G. Estabilidade de formulações cosméticas. International Journal of Pharmaceutical Compounding, v. 3, n. 4, p. 154-156, 2001.

MITSCHKA, P. Simple conversion of Brookfield RVT: readings into viscosity functions. Rheologica Acta, v. 21, n. 2, p. 207-209, 1982.

OLIVEIRA, J.M.D. Aplicação do corante extraído do jenipapo em revestimento orgânico de superfície. São Paulo, 1997. 184p. Dissertação (Mestrado em Eng. Materiais) Escola de Engenharia, Universidade Presbiteriana Mackenzie, São Paulo, 1997. 
PELEGRINE, D.H.; VIDAL, J.R.M.B.; GASPARETTO, C.A. Estudo da viscosidade aparente das polpas de manga (Keitt) e abacaxi (Pérola). Campinas, Ciência e Tecnologia de Alimentos. v. 20, n.1, 2000.

SISKO, A.W. Flow of lubricating greases. Industrial Engineering and Chemistry, v. 50, p. 1789-1792, 1958.

STATISTICA. Data analysis software system, version 7. www.statsoft.com. StatSoft, Inc., 2004. 